\title{
DISTRIBUCION Y ABUNDANCIA DE LARVAS DE EMERITA ANALOGA (STIMPSON 1857) (DECAPODA, ANOMURA) EN LA ZONA DE COQUIMBO Y CALDERA, CHILE
}

\section{DISTRIBUTION AND ABUNDANCE OF EMERITA ANALOGA (STIMPSON 1857) (DECAPODA, ANOMURA) LARVAE IN THE COQUIMBO BAY SYSTEM AND THE COASTAL AREA OF CALDERA, CHILE}

\author{
Eduardo Flores y Armando Mujica \\ Facultad de Ciencias del Mar, Universidad Católica del Norte. Casilla 117, Coquimbo Chile \\ E-mail:eduardofloresb@gmail.com; amujica@ucn.cl
}

\begin{abstract}
RESUMEN
En octubre ( 5 al 26) y diciembre ( 17 al 24) de 2005 se tomaron muestras zooplanctónicas en 40 estaciones, en las zonas de Coquimbo (29 $\left.21^{\prime}-30^{\circ} 15^{\prime} \mathrm{S}\right)$ y Caldera $\left(26^{\circ} 46^{\prime}-27^{\circ} 08^{\prime}\right.$ S) (Proyecto FIP N $\left.{ }^{\circ} 2004-45\right)$. De ellas se separó la totalidad de larvas de Emerita analoga, determinándose sus estados de desarrollo, abundancia relativa, dominancia numérica y frecuencia de ocurrencia. La distribución segregada de los diferentes estados de desarrollo en las dos épocas y áreas muestreadas y su relación con la ubicación de las playas de arena en que viven los adultos, permite inferir la dispersión de las larvas. Se relaciona la distribución y abundancia de las larvas con las posibles zonas de eclosión. Las primeras zoeas fueron más abundantes en ambas zonas, predominando en las estaciones costeras de la zona de Coquimbo, aumentando la abundancia de los estados de desarrollo más avanzados en las estaciones más distante de la costa. En la zona de Caldera, estas larvas fueron menos abundantes, predominando las de desarrollo avanzado en octubre, mientras que en diciembre al igual que en Coquimbo predominaron las primeras zoeas en las estaciones costeras. Las zoeas 1, 2 y 5 fueron más abundantes cerca de las playas de arena. Las zoeas 3 y 4 fueron las de mayor dispersión y distante de estas playas, mientras que las megalopas se encontraron en estaciones de distancia intermedia de la costa.
\end{abstract}

Palabras Clave: Dispersión, Emerita analoga, estadios larvales.

\section{ABSTRACT}

In October $\left(5-26^{\text {th }}\right)$ and December $\left(17-24^{\text {th }}\right)$ of 2005 , zooplankton samples were taken from 40 stations in the Coquimbo bay $\left(29^{\circ} 21^{\prime}-30^{\circ} 15^{\prime} \mathrm{S}\right)$ and the coastal area of Caldera $\left(26^{\circ} 46^{\prime}-27^{\circ} 08^{\prime} \mathrm{S}\right)$ (FIP Project $\mathrm{N}^{\circ}$ 2004-45). All Emerita analoga larvae were taken from the samples and their developmental stages, relative abundances, numerical dominance and frequency of occurrence were determined. The segregated distribution of different larval stages observed in the two sampling periods and locations, and their relationship with the bathymetric distribution of adults on sandy beaches, allowed determining the dispersion of this species. The distribution and abundance of the larvae are related to the spawning possible areas. The early stages were observed predominantly at stations in the coastal area of Coquimbo, while more developed stages were seen in the open ocean. The highest abundances of megalopas were observed in the northern area. In both areas the early stages were most abundant and in Caldera the larvae of this species were less abundant than in Coquimbo. Differences in developmental stage composition between the two sampling seasons were detected. In October the more advanced stages were dominant, while in December a similar trend to the one described for the Coquimbo bay system was observed. Considering the distance from the nearest sandy beaches; zoea 1 and 2 were the most abundant in closer ranges, zoea 3 and 4 showed maximum dispersion from the sandy beaches and zoea 5 and megalopas were again found at shorter distances, demonstrating their return to the sandy beaches in the coastal area.

KEYwORDs: Dispersion, Emerita analoga, larval stages. 


\section{INTRODUCCION}

La primera etapa del ciclo de vida de numerosos invertebrados marinos bentónicos implica la liberación de larvas planctónicas (DiBacco \& Levin 2000). Se han descrito dos procesos que tienen estas larvas en el pélagos: la dispersión, que es el proceso dinámico al que están sometidas las larvas que son exportadas del bentos al pélagos, en el cual permanecen hasta su metamorfosis, para retornar al sustrato como juveniles; y la retención larval, que es el proceso por el cual las larvas tienen conductas que les permite aprovechar corrientes, mareas u otros eventos oceanográficos, que las lleven de retorno a los lugares desde donde fueron eclosionadas o mantenerse cercanas a éstos (Gil 1988).

Las poblaciones de invertebrados bentónicos que tienen larvas planctónicas sujetas a dispersión, constituyen metapoblaciones afectas a factores oceanográficos como las corrientes, eventos localizados (eddies, surgencias) y macroeventos como El Niño (Escribano et al. 2002, Ortiz \& Stotz 2003). Estos factores oceanográficos influyen directamente en el asentamiento y reclutamiento de las larvas meroplanctónicas y por ende en la abundancia y distribución de las poblaciones adultas (Palma et al. 2006).

Factores oceanográficos físicos como los nombrados anteriormente son importantes de considerar en el conocimiento de la dinámica de las poblaciones pelágicas o bentónicas con larvas meroplanctónicas.

Uno de los crustáceos decápodos bentónicos dominantes en las playas de arena de las costas central y norte de Chile es Emerita analoga (Stimpson 1857), conocido como pulga de mar (Contreras et al. 1999). Este anomuro perteneciente a la familia Hippidae, forma agregaciones en la zona de lavado (swash zone) de las playas. Es un animal filtrador que aprovecha la corriente producida por el oleaje en las playas de arena (Barnes \& Wenner 1968). Se ha determinado que es más abundante en playas disipativas que en playas reflectivas, ya que le favorece el perfil plano de la playa y las arenas finas (Jaramillo et al. 2000). Se distribuye desde Alaska $\left(58^{\circ} \mathrm{N}\right)$, hasta el extremo sur de Chile $\left(55^{\circ} \mathrm{S}\right)$, excepto en las regiones tropicales con temperaturas sobre los $20^{\circ} \mathrm{C}$ (Contreras et al. 1999, Jaramillo et al. 2000). Su rango batimétrico varía entre 0 y $3 \mathrm{~m}$.

Esta especie tiene desarrollo indirecto, con huevos portados por las hembras en sus pleópodos, incubación que en condiciones de laboratorio demora entre 29 y 32 días (Barnes \& Wenner 1968, Contreras et al. 1999). A la eclosión se suceden cinco larvas zoeas, hasta llegar al estado de megalopa, con 3 a 5 meses de vida planctónica (Barnes \& Wenner 1968, Sorte et al. 2001). Luego los juveniles se asientan y maduran sexualmente en las playas de arena. Las hembras de $E$. analoga se pueden encontrar ovígeras durante todo el año, con moda durante la primavera e inicios del verano (Contreras et al. 1999).

En el sistema de bahías de la zona de Coquimbo, esta especie se encuentra en playas de arena muy fina a fina (Berrios \& Olivares 1996, Dugan et al. 2000), con granulometría comprendida entre $0,09 \mathrm{~mm}$ y $3,24 \mathrm{~mm}$. Retamal (2000) señala que esta especie habita playas arenosas de granulometría gruesa y altamente oxigenadas.

La distribución batimétrica, características del sustrato en el que se encuentran los adultos de la especie, existencia de playas arenosas en la zona de Coquimbo y Caldera y época reproductiva de la especie, son antecedentes que sustentan el análisis que se efectúa de muestras planctónicas en la época de máximas concentraciones de las larvas de E. analoga, lo que relacionado con la dinámica oceanográfica y la abundancia y distribución de los diferentes estados de desarrollo, permiten conocer aspectos relacionados con la dispersión y retención larval de la especie.

\section{MATERIALES Y METODOS}

En octubre (5 al 26) y diciembre (17 al 24) del año 2005 se efectuaron muestreos de zooplancton en el sistema de bahías de Coquimbo $\left(29^{\circ} 21^{\prime}-30^{\circ} 15^{\prime}\right.$, S) y la zona nerítica de Caldera $\left(26^{\circ} 46^{\prime}-27^{\circ} 08^{\prime}\right.$ S), obteniéndose un total de 160 muestras en 80 estaciones, como parte del proyecto FIP $\mathrm{N}^{\circ}$ 2004-45 "Determinación de las zonas y épocas de reclutamiento de camarón nailon, langostino amarillo y langostino colorado entre la III y IV Región" (Acuña et al. 2007).

Se efectuaron 19 estaciones oceanográficas en la zona comprendida entre bahía Choros (29 21' S) y bahía Tongoy $\left(30^{\circ} 15^{\prime} \mathrm{S}\right)$ y hasta una distancia máxima de $16 \mathrm{mn}$ de la costa (zona de Coquimbo, Fig. 1A) y 21 estaciones entre punta Obispo (26 $46^{\circ}$ S) y bahía Inglesa $\left(27^{\circ} 08^{\prime} \mathrm{S}\right)$, entre la costa y $8 \mathrm{mn}$ de ella (zona de Caldera, Fig. 1B).

Las muestras fueron obtenidas entre la superficie y 


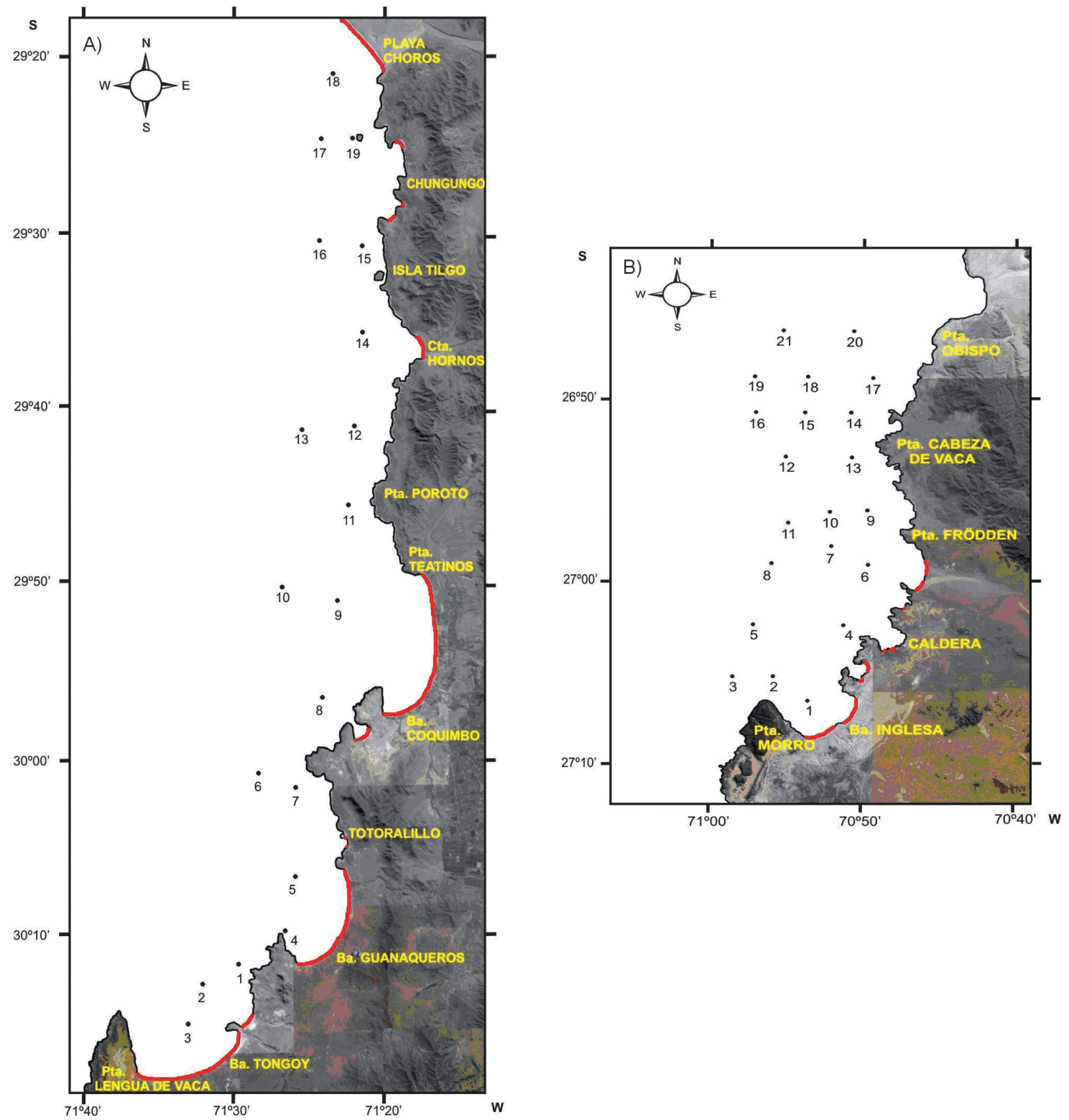

FiguRA. 1. Ubicación de estaciones de muestreo zooplanctónico. A: Zona de Coquimbo; B: Zona de Caldera. Se destacan en rojo las principales playas de arenas de cada zona.

FIGURE 1. Location of zooplankton sampling stations. A: Coquimbo area; B: Caldera area. In red the main sandy beaches in each area are indicated.

$200 \mathrm{~m}$ de profundidad mediante arrastres oblicuos efectuados con redes Bongo cilindro-cónica, de 59 $\mathrm{cm}$ de diámetro de boca, $300 \mu \mathrm{m}$ de abertura de malla y provista de flujómetro.

Las muestras fueron preservadas en solución de formaldehído ( $5 \%$ ) en agua de mar. De ellas se extrajeron las larvas de E. analoga, las que fueron clasificadas por estado de desarrollo según lo descrito por Johnson \& Lewis (1942).

La abundancia relativa del total de larvas de la especie y en cada estado de desarrollo se expresó como número de larvas en $100 \mathrm{~m}^{3}$ de agua filtrada. La dominancia numérica se expresó como el porcentaje de cada estado de desarrollo respecto 
del total de larvas de cada crucero. La constancia o frecuencia de ocurrencia se determinó como el porcentaje de estaciones con presencia de larvas o estado de desarrollo de la especie, respecto del total de estaciones efectuadas.

Para determinar el posible desplazamiento de las larvas desde las playas de arenas en las que viven ejemplares adultos de la especie, se determinó la distancia entre las estaciones y las playas de arenas más cercanas ubicadas al este y sudeste de cada estación.

\section{RESULTADOS}

Entre las larvas capturadas se reconocieron los cinco estados zoea descritos por Johnson \& Lewis (1942), además de un estado megalopa. El 85,4 \% de ellas fueron capturadas en la zona de Coquimbo y sólo el 14,6\% en la zona de Caldera. En la zona de Coquimbo se capturó similar abundancia de larvas en octubre y en diciembre (746 y 763 larvas $\left.100 \mathrm{~m}^{-3}\right)$, mientras que en la zona de Caldera, estas larvas fueron más abundantes en diciembre (142 larvas $\left.100 \mathrm{~m}^{-3}\right)$ que en octubre (117 larvas 100 $\left.\mathrm{m}^{-3}\right)$.

En la zona de Coquimbo, las larvas de esta especie se encontraron en todas las estaciones en ambas épocas de muestreo. En la zona de Caldera se encontraron en el $80,0 \%$ de las estaciones en octubre y en el 90,5\% en diciembre.

En la zona de Coquimbo, en octubre, la mayor abundancia se encontró en la estación 17, frente a Chungungo (158 larvas $100 \mathrm{~m}^{-3}$ ), donde predominaron las zoeas 1 , las cuales fueron capturadas en todas las estaciones de este crucero (100\% de ocurrencia). Las zoeas 2 también tuvieron amplia distribución (Tabla I), aunque fueron más abundantes en la parte norte de esta zona, mientras que las zoeas 3 y 4 tuvieron presencia muy localizada (Fig. 2a) y fueron poco abundantes. A diferencia de esto, las zoeas 5 fueron más abundante que los dos estados precedentes, con amplia distribución y máximos en la parte norte de

TABla I. Abundancia, dominancia numérica y frecuencia de estados de desarrollo de larvas de E. analoga en zonas y época de muestreo.

TABLE I. Abundance, numerical dominance and frequency of E. analoga larvae developmental stages, in sampling areas and seasons.

\begin{tabular}{|c|c|c|c|c|c|c|c|}
\hline \multirow[b]{2}{*}{ Zona } & \multirow[b]{2}{*}{ Estadio } & \multicolumn{3}{|c|}{ Octubre } & \multicolumn{3}{|c|}{ Diciembre } \\
\hline & & $\begin{array}{l}\text { Abundancia } \\
\left(\mathrm{N}^{\circ} 100 \mathrm{~m}^{-3}\right)\end{array}$ & $\begin{array}{c}\text { Dominancia } \\
(\%)\end{array}$ & $\begin{array}{c}\text { Frecuencia } \\
(\%)\end{array}$ & $\begin{array}{l}\text { Abundancia } \\
\left(\mathrm{N}^{\circ} 100 \mathrm{~m}^{-3}\right)\end{array}$ & $\begin{array}{c}\text { Dominancia } \\
(\%)\end{array}$ & $\begin{array}{c}\text { Frecuencia } \\
\qquad(\%)\end{array}$ \\
\hline \multirow{6}{*}{ Coquimbo } & $\mathrm{Z} 1$ & 361 & 48,4 & 100 & 662 & 86,8 & 78,9 \\
\hline & $\mathrm{Z} 2$ & 160 & 21,4 & 68,4 & 17 & 2,2 & 52,6 \\
\hline & $\mathrm{Z3}$ & 9 & 1,2 & 26,3 & 7 & 0,9 & 21,1 \\
\hline & $\mathrm{Z} 4$ & 2 & 0,3 & 10,5 & 21 & 2,8 & 57,9 \\
\hline & $\mathrm{Z} 5$ & 214 & 28,7 & 89,5 & 51 & 6,7 & 73,7 \\
\hline & M1 & & & & 5 & 0,7 & 15,8 \\
\hline \multirow{5}{*}{ Caldera } & $\mathrm{Z} 1$ & 8 & 6,8 & 23,8 & 79 & 55,6 & 38,1 \\
\hline & $\mathrm{Z} 2$ & 0 & 0,0 & 0 & 5 & 3,5 & 23,8 \\
\hline & $\mathrm{Z3}$ & 14 & 12,0 & 23,8 & 7 & 4,9 & 38,1 \\
\hline & $\mathrm{Z} 4$ & 39 & 33,3 & 66,7 & 25 & 17,6 & 66,7 \\
\hline & $\mathrm{Z} 5$ & 56 & 47,9 & 57,1 & 26 & 18,3 & 57,1 \\
\hline
\end{tabular}




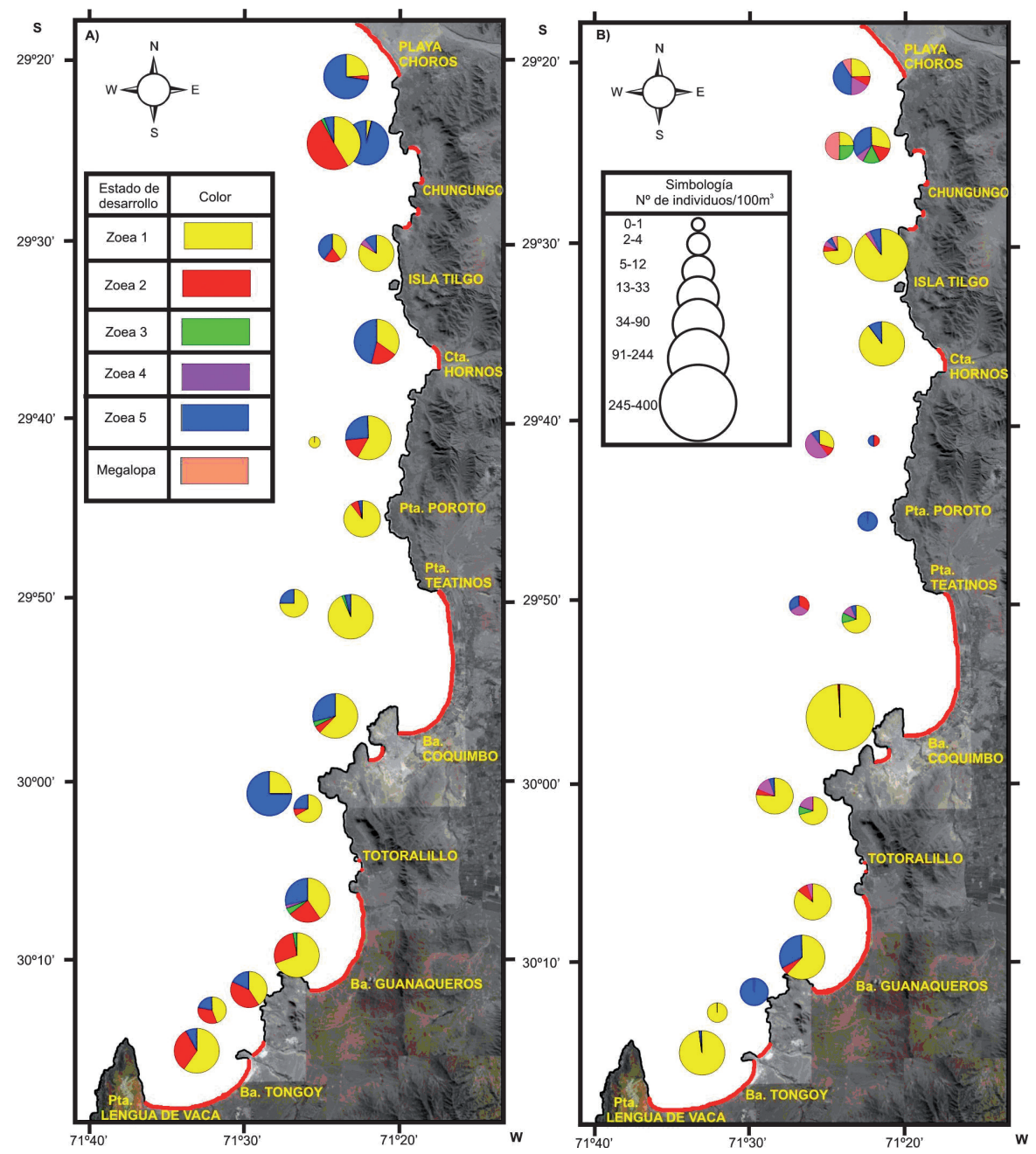

FiguRA 2. Distribución y abundancia de larvas de E. analoga por estados de desarrollo en la zona de Coquimbo, A) octubre y B) diciembre de 2005.

FigurE 2. Distribution and abundance per development state of E. analoga larvae in Coquimbo area. A) October. B) December 2005.

esta zona de estudio (Est. 18 y 19), próximas a la playa Choros (Fig. 2A).

En diciembre, en esta zona, la mayor abundancia (402 larvas $100 \mathrm{~m}^{-3}$ ) se encontró en una estación ubicada frente a la bahía Herradura de Guayacán, en la que predominaron ampliamente las zoeas 1 (Fig. 2B), que al igual que en el muestreo anterior, tuvieron amplia distribución y fue el estado de desarrollo de mayor dominancia numérica, mientras que los otros estados de desarrollo fueron poco abundantes (Tabla I). A diferencia del muestreo anterior, en esta oportunidad, las zoeas 1 no se encontraron en todas las estaciones, pero representaron más del $85 \%$ del total de larvas, mientras que los restantes estados de desarrollo tuvieron dominancias inferiores al $7 \%$. Entre estos estados se destaca la presencia de megalopas en tres estaciones en la parte norte del área de estudio y la frecuencia de ocurrencia de las zoeas 5 que fue similar a las de las zoeas 1 (Tabla I).

En el muestreo de octubre, en la zona de Caldera, se capturaron larvas de esta especie en la mayoría de 


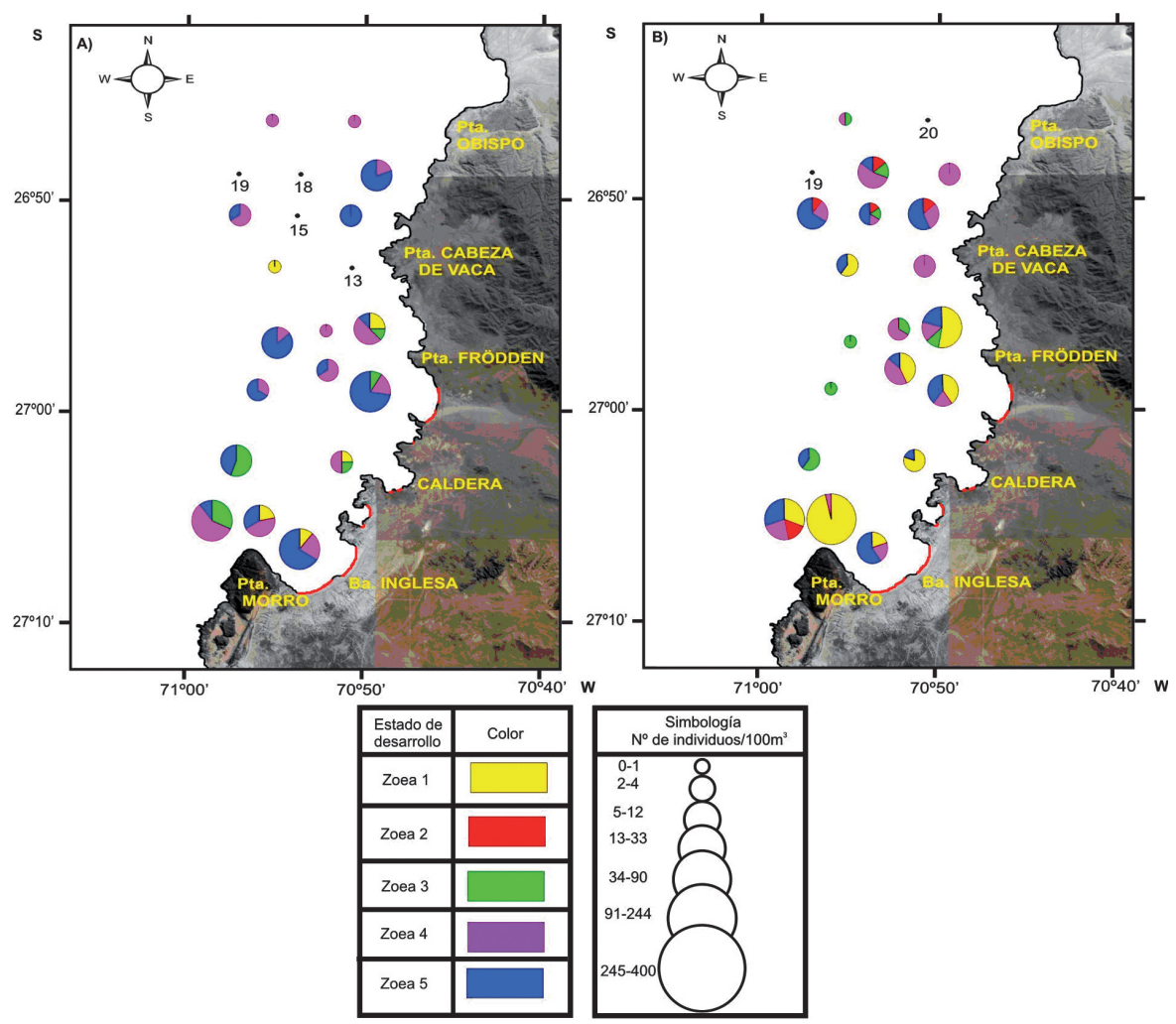

Figura 3. Distribución y abundancia de larvas de E. analoga por estado de desarrollo en la zona de Caldera, A) durante octubre y B) durante diciembre de 2005.

FIgURE 3. Distribution and abundance per development state of E. analoga larvae in Caldera area. A) October. B) December 2005.

las estaciones, en baja abundancia y principalmente en la parte sur del área de muestreo (Fig. 3A). Las mayores abundancias fluctuaron entre 16 y 18 larvas $100 \mathrm{~m}^{-3}$ (Est. 1, 3 y 6). En esta oportunidad, predominaron las zoeas 4 y 5 , mientras que no se capturaron zoeas 2 (Tabla I).

En diciembre, en esta zona, la mayor abundancia se encontró en una estación ubicada frente a bahía Inglesa (Est. 2), en la que predominaron las zoeas 1. Este estado de desarrollo se encontró preferentemente en las estaciones costeras de la parte sur de esta zona (Fig. 3B). Las zoeas 2 y 3 tuvieron baja abundancia relativa y se capturaron principalmente en la parte norte las primeras y en estaciones oceánicas las otras. Las zoeas 4 y 5 tuvieron amplia distribución y fueron más abundantes que las zoeas 2 y 3 (Fig. 3B).

Las mayores concentraciones de zoeas 1 se encontraron en estaciones a no más de $7 \mathrm{mn}$ de las playas más cercanas ubicadas al este o sudeste; aunque en la zona de Coquimbo se encontraron larvas en este estadio en mínimas concentraciones hasta $13 \mathrm{mn}$, de las playas antes referidas (Figs. 4 y 5). Las zoeas 2 fueron más abundantes en las estaciones cercanas a las playas de arena en la zona de Coquimbo, mientras que en la zona de Caldera fueron más abundantes en las estaciones de distancia intermedia de las playas de arena más cercana (entre 7 y $11 \mathrm{mn}$ ), al igual que la distribución de las megalopas, que se capturaron entre 4 y $10 \mathrm{mn}$ de las playas de arena más cercana en la zona de Coquimbo (Figs. 4 y 5).

Las mayores abundancias de las zoeas 5 , que se encontraron próximas a las playas de arena, tuvieron una tendencia similar a la de las zoeas 1 , mientras que las zoeas 3 y 4 , que tuvieron abundancias menores que los estadios precedentes, se distribuyeron tanto en estaciones próximas a las playas de arenas como en las más distantes, encontrándose hasta $15 \mathrm{mn}$ de ellas (Figs. 4 y 5). 


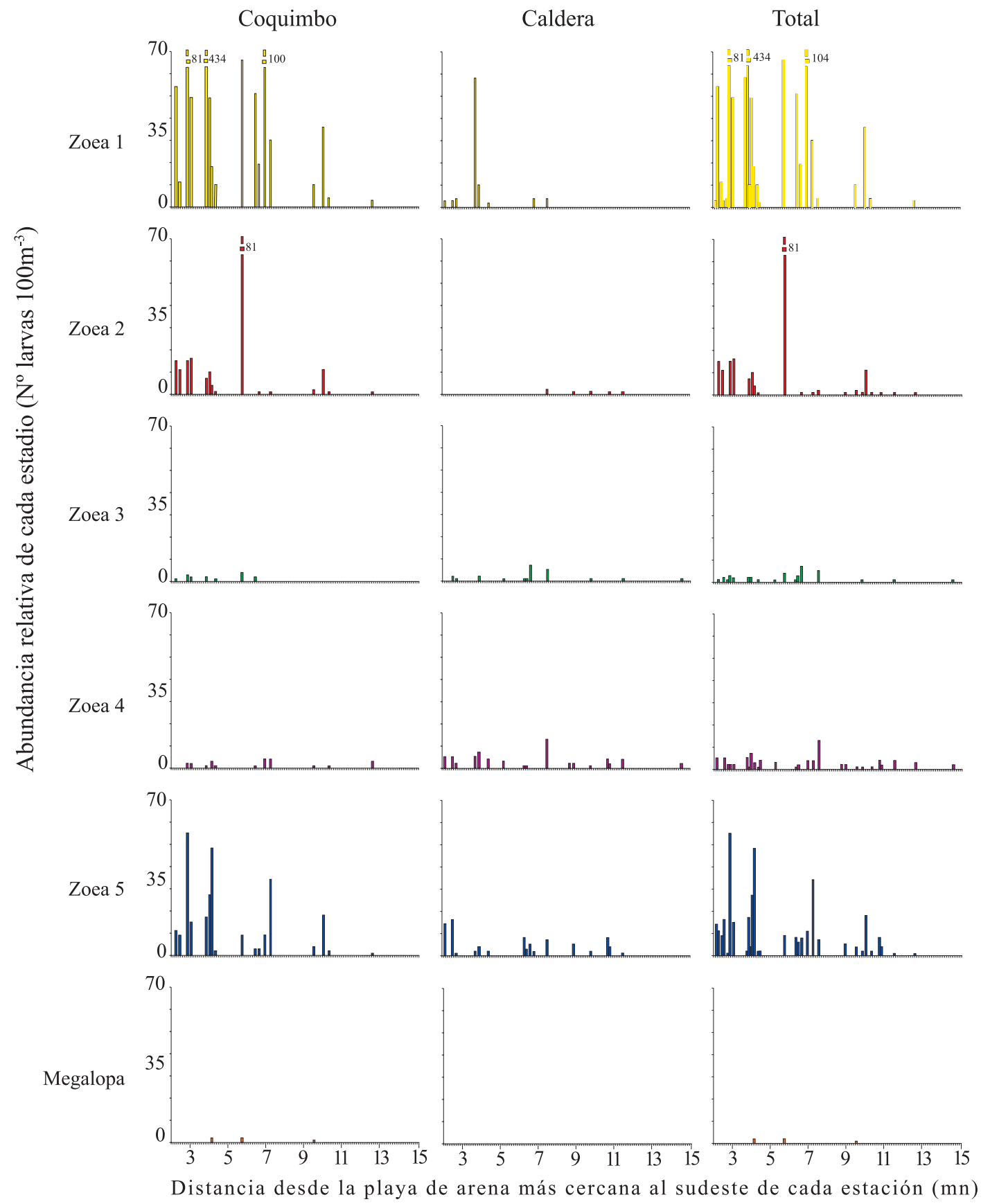

FIgURA 4. Abundancia relativa de estados de desarrollo en estaciones ordenadas de acuerdo a la distancia desde la playa de arena más cercana ubicada al este o sudeste.

FIGURE 4. Relative abundance of each developmental stage in stations arranged according to the distance from the nearest sandy beach located to the east or southeast. 


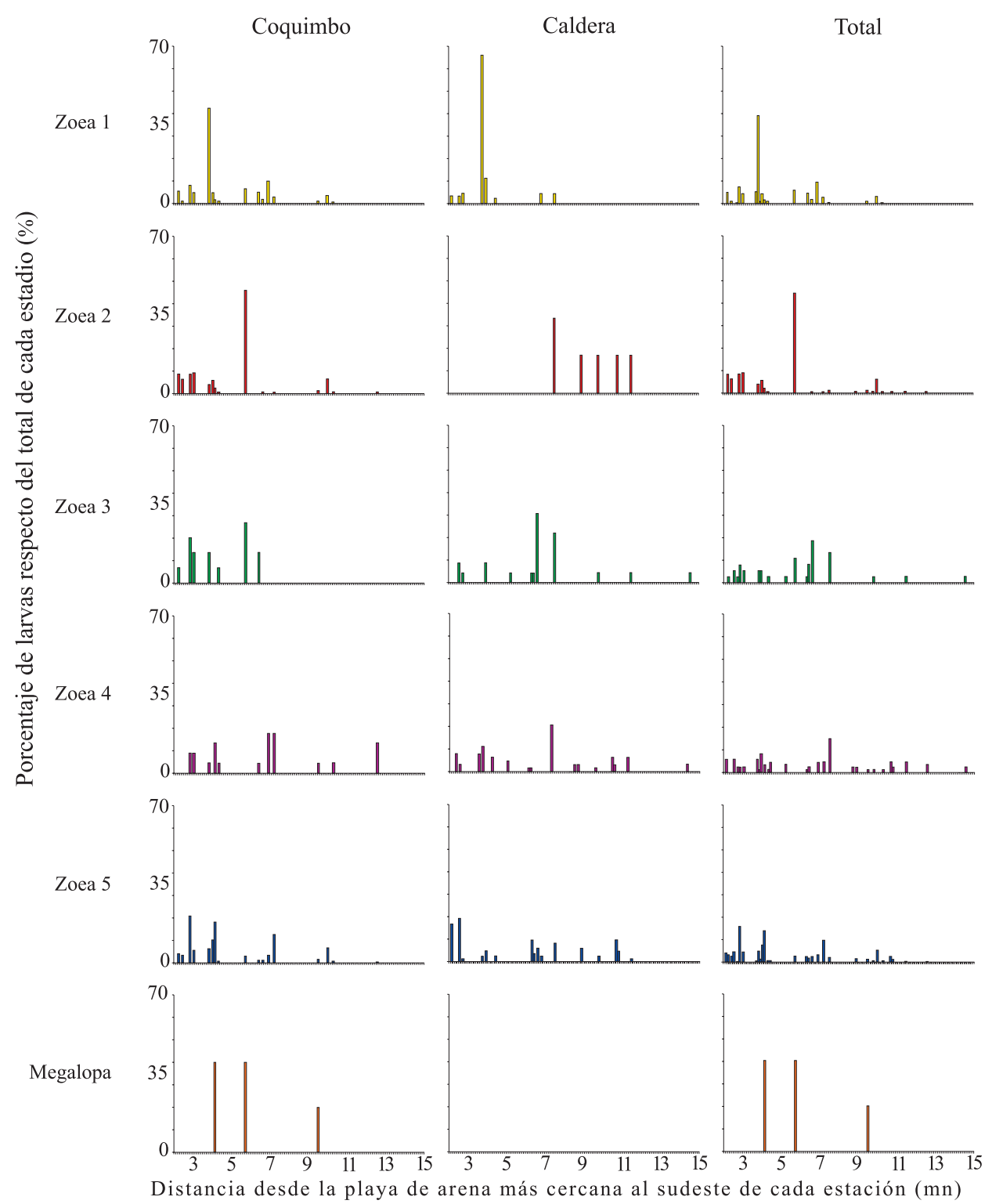

FIGURA 5. Abundancia de larvas (\% de cada estado de desarrollo) en estaciones ordenadas de acuerdo a la distancia desde la playa de arena más cercana ubicadas al este o sudeste.

FIGURE 5. Larvae abundance (\% each developmental stage) in stations arranged according to the distance from the nearest sandy beach located to the east or southeast.

\section{DISCUSION}

Las mayores abundancias de zoeas 1 y 2 en las proximidades de la costa, que no han permanecido el tiempo suficiente en el plancton para distanciarse del lugar de eclosión, corresponde con la distribución de las poblaciones de adultos en playas arenosas. Además, el aumento de la abundancia de las zoeas 1 , detectada en verano respecto de la primavera, coincide con la época de máxima actividad reproductiva de poblaciones de E. analoga en las costas del norte y centro de Chile (Contreras et al. 1999).

La mayor abundancia de estas larvas en la zona de Coquimbo, respecto de Caldera, se debería al menor número y áreas de playas de arenas que existen en esta última zona, en cuya parte norte la mayoría de las playas son de bolones o de granulometría que no 
permite la existencia de poblaciones de adultos de la especie, lo que fue corroborado con la presencia de larvas de estados tempranos de desarrollo, frente a Caldera y Bahía Inglesa, que son las playas de arena de mayor extensión de esta zona de muestreo.

El predominio de los estados de desarrollo intermedios (zoeas 3 y 4), en las estaciones más alejadas de las playas de arenas, sería producto del tiempo de permanencia de éstas en el plancton y los procesos advectivos que las alejarían de los lugares de eclosión, llegando a distanciarse más de $14 \mathrm{mn}$ desde la playa de arena más cercana.

Según Acuña et al. (1989), el foco de surgencia que se encuentra frente al Parque Nacional Fray Jorge $\left(31^{\circ} \mathrm{S}\right)$ tiene influencia en todo el sistema de bahías de Coquimbo, lo que transportaría mar adentro a las larvas eclosionadas próximas a la costa, lo que explicaría la mayor abundancia de los estados intermedios alejados de la costa.

Por otra parte, la presencia de todos los estados zoea y las megalopas en las estaciones cercanas a playa Choros estaría determinada por la dinámica oceanográfica local, que es producto de la existencia de islas que permiten la confluencia de aguas provenientes del sur y oeste, generando focos de surgencia (Acuña et al. 2007), en lo que se ha señalado como un lugar de retención larval (Mujica \& Acuña 2009).

Las zoeas 5, al igual que los estados de desarrollo tempranos, tuvieron una distribución principalmente costera, aunque más concentrada en la parte norte de esta área de estudio, lo que estaría asociado a la deriva que experimentan las larvas, producto del efecto de la corriente Chile-Perú (corriente de Humboldt), la cual fluye de sur a norte y es la predominante en ambas áreas de estudio (Escribano et al. 2002). Al respecto, cabe mencionar que en el sistema de bahías de Coquimbo se han efectuado diversos estudios que señalan un flujo con dirección norte, producto de dos giros causados por la circulación geostrófica y la componente diurna de mareas, permitiendo que aguas provenientes desde la bahía Tongoy lleguen a las bahías al norte de ésta (Acuña et al. 1989). Este efecto oceanográfico es de carácter estacional, el cual en primavera (octubre) permite la existencia de dos regiones de flujos hacia el norte, que son superficiales y alcanzan hasta los $50 \mathrm{~m}$. Uno costero y otro más alejado de la costa, los que están separados por un flujo menor con dirección sur, que ocurre en profundidades de 100 a 200 m (Moraga et al. 1994).
Esto permite suponer que componentes planctónicos ubicados a diversas profundidades y distancia de la costa tendrían desplazamientos diferentes, lo cual explicaría la deriva y retención de componentes tanto meroplanctónicos (langostino colorado y amarillo, camarón nailon, anchoveta y estomatópodos) como holoplanctónicos (salpas, quetognatos y eufáusidos), en estos sistemas de bahías y que han sido reportadas por diversos autores (Acuña et al. 1995, Miranda 1995, Ríos 1996, Acuña et al. 1997a, Acuña et al. 1997b, Fuentes 1999, Silva 1999, Ottone 2006, Quiroz et al.2006, Acuña et al. 2007, Gallardo 2007, González 2007, Mujica \& Acuña 2009).

La circulación interna de la bahía de Caldera se ha definido como de tipo estuarina, con estratificación de la columna de agua (Ogalde 2000). A diferencia de la zona de Coquimbo, en esta zona se conoce poco la dinámica oceanográfica entre las bahías, aunque en general se puede señalar que ésta es muy parecida a la de Coquimbo (Escribano et al. 2002). Sin embargo, basado en la abundancia y distribución de los estados de desarrollo larvales detectados en esta oportunidad, es posible inferir que frente a Bahía Inglesa existirían efectos oceanográficos (giros, remolinos, sombra de surgencia, o entradas de aguas desde el oeste) que permiten la retención larval. Concordante con esto, la abundancias de estados de desarrollo intermedios de E. analoga, encontrados cerca de esta bahía, coincide con lo encontrado por Acuña et al. (2007) y Mujica \& Acuña (2009); aunque no se descarta que ellas provengan de playas de arenas ubicadas al sur de la zona muestreada como son Bahía Copiapó ( $\left.27^{\circ} 20^{\prime} \mathrm{S}\right)$ y Bahía Cisne $\left(27^{\circ} 14^{\prime} \mathrm{S}\right)$, las cuales tienen granulometría apta para el desarrollo de poblaciones adultas.

Las poblaciones de adultos de E. analoga en las playas arenosas de las zonas estudiadas y la dinámica oceanográfica de ellas, sumado a la distribución segregada de los diferentes estados de desarrollo de las larvas en áreas adyacentes, sugiere la dispersión y retorno de ellas a zonas costeras aptas para su asentamiento, lo que debería estar relacionado con la migración vertical ontogénica descrita para las larvas de crustáceos (Lindley 1986, Shanks 1986, Queiroga 1998), en la que los primeros estados de desarrollo se encontrarían en el estrato superficial y los más avanzados en estratos más profundos, hipótesis que debería ser comprobada por estudios que contemplen muestreos estratificados, en especial si se considera que ella contradice parcialmente lo postulado por Barnes \& Wenner (1968), quienes señalan que las 
larvas de E. analoga sólo se encuentran en estratos superficiales de la columna de agua.

Por último, se estima que el muestreo estratificado del plancton, en conjunto con el uso de marcadores genéticos para establecer el grado de parentesco entre poblaciones, permitiría comprobar con mayor precisión la deriva y procedencia de larvas de poblaciones de crustáceos bentónicos definidos.

\section{AGRADECIMIENTOS}

Las muestras zooplanctónicas y los datos oceanográficos del presente estudio fueron obtenidas en el desarrollo del proyecto FIP $N^{\circ}$ 2004-45. Los autores agradecen al profesor Julio Moraga Opazo, quien facilitó la información oceanográfica física. También agradecen la revisión crítica y aportes al documento que efectuaron Sarah E. Allan y María Luisa Nava.

\section{BIBLIOGRAFIA}

Acuña, E., Moraga, J. \& Uribe, E. 1989. La zona de Coquimbo: Un sistema nerítico de alta productividad. Revista del Pacífico Sur (Número especial), pp. 145-157.

Acuña, E., Arancibia, H., Mujica, A., Brokordt, K. \& Gaymer, C. 1995. Estudio biológico-pesquero del langostino amarillo (Cervimunida johni) en la III y IV Región, mediante el uso de la flota arrastrera con base en Coquimbo. Informe Final Proyecto U.C. del Norte-Sede Coquimbo/Instituto de Investigación Pesquera VIII Región/Empresas Pesqueras de Coquimbo, 107 pp.

Acuña, E., Arancibia, H., Mujica, A., Cid, L. \& Roa, R. 1997a. Análisis de la pesquería y evaluación indirecta del stock de langostino amarillo en la III y IV Regiones. Informes Técnicos FIP. FIP/ IT No 96-08, 153 pp.

Acuña, E., Arancibia, H., Mujica, A., Cid, L., Roa, R. \& Winkler, F. 1997b. Análisis de la pesquería y evaluación indirecta del stock de camarón nailon (III y VIII Regiones). Informes Técnicos FIP. FIP/ IT $\mathrm{N}^{\circ}$ 95-06, $211 \mathrm{pp}$.

Acuña, E., Andrade, M., Cubillos, L., Arancibia, H., Moraga, J., Mujica, A., Berrios, M., Lancelloti, D., Villarroel, J.C., Haye, P. \& Stotz, W. 2007. Determinación de zonas y épocas de reclutamiento de camarón nailon, langostino amarillo y langostino colorado en las regiones III y IV, año 2007. Informes Técnicos FIP. FIP/IT N ${ }^{\circ}$ 2004-45, 345 pp.

BARnes, N. \& Wenner, A. 1968. Seasonal Variation in the Sand Crab Emerita analoga (Decapoda, Hippidae) in the Santa Barbara Area of California. Limnology and Oceanography.13: 465-475.

Berrios, M. \& Olivares, J. 1996. Caracterización Granulométrica y Contenido de Carbono Orgánico de los Sedimentos Marinos Superficiales, en el Sistema de Bahías de la IV Región. Coquimbo. Ciencia y Tecnología del Mar. 19: 37-45.

Contreras, H., Defeo, O. \& Jaramillo, E. 1999. Life History of Emerita analoga (Stimpson) (Anomura, Hippidae) in a Sandy Beach of South Central Chile. Estuarine, Coastal and Shelf Science. 48: 101-112.

DiBacco, C. \& Levin, L. 2000. Development and application of elemental fingerprinting to track the dispersal of marine invertebrate larvae. Limnology and Oceanography. 45: 871-880.

Dugan, J., Hubbarda, D. \& Lastra, M. 2000. Burrowing abilities and swash behavior of three crabs, Emerita analoga Stimpson, Blepharipoda occidentalis Randall, and Lepidopa californica Efford (Anomura, Hippoidea), of exposed sandy beaches. Experimental Marine Biology and Ecology. 255: 229-245.

Escribano R., Marín, V., Hidalgo, P. \& Olivares, G. 2002. Physical-Biological interactions in the Pelagic Ecosystem of the Nearshore Zone of the Northern Humboldt Current System. En: The Oceanography and Ecology of the nearshore and bays in Chile. (Eds Castilla, J.C. \& J.L. Largier), Ediciones Universidad Católica de Chile, Santiago, Chile. pp. 145-175.

Fuentes, P. 1999. Composición, distribución y abundancia de eufáusidos (Crustacea: Malacostraca) en la zona comprendida entre Punta Damas y Punta Lengua de Vaca IV Región, Chile. Tesis para optar al grado de Biólogo Marino. Facultad de Ciencias del Mar. Universidad Católica del Norte. Coquimbo, Chile.

Gallardo, M. 2007. Descripción, distribución y abundancia de larvas de Mursia gaudichaudi $(\mathrm{H}$. Milne Edwards, 1837) (Decapoda, Brachyura, Calappidae) en la III y IV Región (26 $45^{\circ}-30^{\circ}$ $10^{\prime}$ S), Chile. Tesis para optar al grado de Biólogo Marino. Facultad de Ciencias del Mar. Universidad Católica del Norte. Coquimbo, Chile.

GIL, R. 1988. Dispersión o retención: el problema de las larvas de Cryphiops caementarius (Crustacea: Palaemonidae) en el estuario del río Limarí. IV Región. Tesis para optar al grado de Biólogo Marino. Facultad de Ciencias del Mar. Universidad Católica del Norte. Coquimbo, Chile.

GonZÁLEZ, A. 2007. Descripción, distribución y abundancia de larvas de estomatópodos (Hoplocarida: Stomatopoda) en la costa de la III y IV Región de Chile $\left(26^{\circ}-30^{\circ} \mathrm{S}\right)$ y su relación con las condiciones oceanográficas. Tesis para optar al grado de Biólogo Marino. Facultad de Ciencias del Mar. Universidad Católica del Norte. Coquimbo, Chile.

Jaramillo, E., Dugan, J. \& Contreras, H. 2000. Abundance, Tidal Movement, Population 
Structure and Burrowing Rate of Emerita analoga (Anomura, Hippidae) at a Dissipative and Reflective Sandy Beach in South Central Chile. Marine Ecology 21: 113-127.

Johnson, M. \& Lewis, W. 1942. Pelagic Larval Stages of the Sand Crabs Emerita analoga (Stimpson), Blepharipoda occidentalis Randall, and Lepidopa myops Stimpson. Biological Bulletin 83: 67-87.

LindLEY, J. A. 1986. Vertical distributions of decapod crustacean larvae and pelagic post-larvae over Great Sole Bank (Celtic Sea) in June 1983. Marine Biology 90: 545-549.

Miranda, O. 1995. Taxonomía, distribución y variación espacio-temporal de la familia Salpidae (Tunicata: Thaliacea), en la zona comprendida entre Pta. Damas y Pta. Lengua de Vaca. IV Región. Chile. Tesis para optar al grado de Biólogo Marino. Facultad de Ciencias del Mar. Universidad Católica del Norte. Coquimbo, Chile.

Moraga, J., Valle-Levinson, A. \& Blanco, J. 1994. Hidrografía y dinámica de la capa superior del océano en la zona costera del pacífico sur oriental $\left(30^{\circ} \mathrm{S}\right)$. Investigaciones Pesqueras (Chile). 38: $55-73$.

MujicA, A. \& Acuña, E. 2009. Larvas de Heterocarpus reedi Bahamonde 1955, Cervimunida johni Porter 1903 y Pleuroncodes monodon H. Milne Edwards 1837 , en la zona de Coquimbo $\left(29^{\circ} 20^{\prime} \mathrm{S}-30^{\circ} 14^{\prime} \mathrm{S}\right)$ y Caldera $\left(26^{\circ} 45^{\prime} \mathrm{S}-27^{\circ} 6^{\prime} \mathrm{S}\right)$, Chile. Revista Biología Marina y Oceanografía (en prensa).

Ogalde, H. 2000. Estudio del intercambio de agua en la bahía de Caldera, III Región. Tesis para optar al grado de Biólogo Marino. Facultad de Ciencias del Mar. Universidad Católica del Norte. Coquimbo, Chile.

Ortiz, M. \& Stotz, W. 2003. Estimación preliminar del número óptimo de muestras para la evaluación del reclutamiento en Mesodesma donacium (Lamarck, 1818) en playas del centro-norte de Chile: Aplicación del análisis de poder. Ciencias Marinas 29: 371-375.

Ottone, N. 2006. Distribución y abundancia de larvas de Engraulis ringens y su relación con predadores zooplanctónicos en la $3^{\mathrm{a}}$ y $4^{\mathrm{a}}$ Región. Tesis para optar al grado de Biólogo Marino. Facultad de Ciencias del Mar. Universidad Católica del Norte,
Coquimbo, Chile.

Palma, A., Pardo, L., Veas, R., Cartes, C., Silva, M., Manríluez, K., Díaz, A., Muñoz, C. \& OJeda, F. 2006. Coastal brachyuran decapods: settlement and recruitment under contrasting coastal geometry conditions. Marine Ecology Progress Series 316: 139-153.

Queiroga, H. 1998. Vertical migration and selective tidal stream transport in the megalopa of the crab Carcinus maenas. Hydrobiologia, 375/376: 137-149.

Quiroz, J., Montenegro, C., Báez, P., Espínola, F., Canales, C., Reyes, H., Magnere, O., Yáñez, O., Tapia, J., Bahamonde, R., Arriagada, G. \& GÁlvez, P. 2006. Dinámica y estructura poblacional del langostino colorado III y IV Regiones. Informes Técnicos FIP. FIP/IT 200541. $340 \mathrm{pp}$.

Retamal, M. 2000. Decápodos de Chile. 1995. World Biodiversity Database CD-ROM Series. ETI-U. de Concepción. Springer-Verlag.

Ríos, J. 1996. Distribución y abundancia espaciotemporal del Phylum Chaetognatha en la zona comprendida entre punta Lengua de Vaca $\left(30^{\circ} 7,8^{\prime}-\mathrm{S}\right)$ y punta Damas $\left(29^{\circ} 15,8^{\prime}-\mathrm{S}\right)$. Tesis para optar al grado de Biólogo Marino. Facultad de Ciencias del Mar. Universidad Católica del Norte. Coquimbo, Chile.

Shanks, A. L. 1986. Vertical migration and cross-shelf dispersal of larval Cancer spp. and Randallia ornata (Crustacea: Brachyura) off the coast of southern California. Marine Biology 92: 189199.

SiLVA, J. 1999. Distribución y abundancia de larvas de langostino amarillo Cervimunida johni (Poster, 1903) y langostino colorado Pleuroncodes monodon (H. Milne Edwards, 1837) frente a la costa de Coquimbo. Tesis para optar al grado de Biólogo Marino. Facultad de Ciencias del Mar. Universidad Católica del Norte. Coquimbo, Chile.

Sorte, C., Peterson, W., Morgan, C. \& Emmertt, R. 2001. Larval dynamics of the sand crab, Emerita analoga, off the central Oregon coast during a strong El Niño period. Journal of Plankton Research 23: 939-944. 